\title{
Caregiver's distress related to the patient's neuropsychiatric symptoms as a function of the care-setting
}

\author{
Laura Lorenzo-López, $\mathrm{PhD}^{\mathrm{a}}$, Carmen de Labra, $\mathrm{PhD}^{\mathrm{b}}$, Ana Maseda, $\mathrm{PhD}^{\mathrm{a}}$, \\ Trinidad Lorenzo, $\mathrm{PhD}^{\mathrm{b}}$, Helena Agrafojo, $\mathrm{PhD}^{\mathrm{a}}$, José Luis Rodríguez-Villamil, \\ $\mathrm{PhD}^{\mathrm{a}}$, Isabel González-Abraldes, $\mathrm{PhD}^{\mathrm{a}}$, José C. Millán-Calenti, PhD ${ }^{\mathrm{a}, \text {, }}$ \\ ${ }^{a}$ Gerontology Research Group, Instituto de Investigación Biomédica de A Coruña (INIBIC), Complexo
Hospitalario Universitario de A Coruña (CHUAC), SERGAS, Universidade da Coruña, 15006 A Coruña,
Spain
${ }_{b}^{b}$ Research, Development and Innovation Department, Gerontological Complex La Milagrosa, Provincial
Association of Pensioners and Retired People (UDP) from A Coruña, A Coruña, Spain
}

\begin{abstract}
Prevalence of neuropsychiatric symptoms (NPS) and correlation between its intensity and caregiver distress were evaluated as a function of the care-setting in a cross-sectional study including 72 patient-caregiver dyads. The Neuropsychiatric Inventory and the Caregiver Distress Scale were administered. The most prevalent symptoms were: in the formal care-setting, agitation/aggression and depression/dysphoria (42.4\%), and in the informal care-setting, aberrant motor behavior (59.0\%). While changes in appetite and eating behaviors, depression/dysphoria and irritability/lability were more prevalent in the formal care-setting ( $p<.0001, p=.011, p=.021$ ), aberrant motor behavior was more prevalent in the informal care-setting $(p=.007)$. NPS were positively correlated with caregiver distress. High patients' scores in hallucinations, sleep and night-time disturbances, anxiety, and aberrant motor behavior were the best predictors of professional caregiver's distress. Agitation/aggression, delusions, disinhibition, apathy/indifference, depression/dysphoria, and elation/euphoria were the best predictors of informal caregiver's distress. Findings may have important clinical implications.
\end{abstract}

Keywords

Institutionalized elderly people; Neuropsychiatric symptoms; Caregiver distress; Care-setting 


\section{Introduction}

The elderly population and the aging process itself are chief concerns in developed and developing countries. The 2015 Ageing Report ${ }^{1}$ showed that, from the beginning of the century, improvements in general living conditions have dramatically increased life expectancy. The prevalence of different pathologies and age-related syndromes increases throughout the aging process. Dementia is a common and serious syndrome characterized by a global cognitive and functional decline, as well as by the presence, in the majority of cases, of neuropsychiatric symptoms (NPS) such as delusions, hallucinations, agitation/aggression, depression/dysphoria, anxiety, elation/euphoria, apathy/indifference, disinhibition, irritability/lability, aberrant motor behavior, sleep and night-time behavior disturbances, and changes in appetite and eating behaviors. ${ }^{2 ;}$ NPS have important implications for patients and their families, as they generate significant stress, not only in the patients but also in their caregivers, as their physical, psychological, and emotional distress increase. ${ }^{4 ; 5 ; 6}$ In fact, a high prevalence of NPS in patients negatively impact quality of life of caregivers. ${ }^{7}$ With these associated problems, informal caregivers may find necessary to take the patient into a day-care center (semi-institutionalization) or even decide on potentially premature institutionalization. ${ }^{8}$ Day care assistance is effective in reducing NPS in dementia patients and in alleviating caregivers' burden. ${ }^{9}$ These possibilities demonstrate two distinct types of scenarios: the support group of "informal caregivers," including family members, friends, and other people who are close to the patient, sometimes paired with formal care services when the option is a day-care center; and a "formal care system" provided by nursing staff when the patient has been institutionalized. One essential characteristic of informal caregiving is the decrease in economic and social costs because the patient is living with his/her family, whilst, on the contrary, formal caregiving by specialists results in a more expensive cost. Despite the particularities of each type of care-setting, and despite the clear difference existing between these two types of support, it is widely accepted that both, informal and formal caregiving, imply a high degree of distress. ${ }^{4 ; 5 ; 6 ; 10}$

Given the relatively high incidence of NPS in each care-setting and the modest efficacy of current strategies, correct identification and evaluation of NPS are a relevant and meaningful clinical target for intervention in dementia care. The present study has the ability to increase our understanding of how to improve non-pharmacological caregiver interventions and reduce the adverse outcomes associated with NPS in both formal and informal care-settings. This is relevant because the association between NPS and caregiver distress may be different as a function of the care context. It is important to note that most of previous studies used the NPI total score as the sum of individual neuropsychiatric symptoms scores. Because this approach does not provide information on specific dimensions, which may have different etiologies, symptoms and prognosis (and interventions/treatments response), and which may differently impact on caregiver distress and wellbeing, we have analyzed single NPS scores in the present study. It has been demonstrated that some individual symptoms may result in more caregiver distress than others. ${ }^{11}$ Further studying individual symptoms is a relevant point, since there is no consistent evidence in the literature about specific or symptom-targeted strategies for individual NPS. By knowing which individual symptoms generate distress and their association with the provision of help (formal vs. informal care context), it could allow developing effective interventions to improve the caregivers' wellbeing.

Bearing all of this in mind, the primary aim of this article was to explore the phenomenon of caregiving distress in a population of formal (professional) and informal caregivers by assessing their likely predictors of distress related to individual patients' NPS, and to test the hypothesis that individual NPS will be different in both care contexts as predictors of caregivers' distress. 


\section{Material and methods}

\section{Participants}

Patients and caregivers were recruited from a Gerontological Complex sited in A Coruña, Spain. The complex has the capacity for 70 semi-institutionalized people in a day care-setting and 64 institutionalized people in a nursing home. We used a purposive/judgmental nonprobability sampling method, being the specific inclusion criteria for the patients: (1) aged 65 or above, (2) having shown at least one of any of the 12 neuropsychiatric assessed symptoms in the previous 4 weeks, and (3) willingness to sign the informed consent form directly or through their legal representative. The inclusion criteria for formal caregivers (paid institutional care at nursinghome) were being the professional providers of assistance to the patients with activities of daily living for at least the previous 6 months, and signing informed written consent for study participation. For family caregivers, the inclusion criteria were being the primary informal caregiver (unpaid care) for at least the previous 6 months (having a significant personal relationship and providing a broad range of assistance to the patient), irrespective of whether the caregiver lives with or separately from the patient, and signing informed written consent. 107 patient-caregiver dyads were enrolled. Of these 107 patients, 67.3\% $(n=72)$ had shown at least one of any of the 12 neuropsychiatric symptoms in the previous 4 weeks. Thus, 72 patientcaregiver dyads (formal care-setting $n=33$; informal care-setting $n=39$ ) were finally included in the study.

\section{Procedure}

The study protocol was approved by the Ethics Committee of the University of A Coruña and the research was conducted in accordance with the ethical standards of the Declaration of Helsinki. Before data collection, all patients (or their legal representatives) and caregivers were informed about the study and provided their informed consent to participate.

\section{Variables and instruments}

A structured sociodemographic questionnaire was designed to record self-reported information on patient's age, gender, and educational level, classified into three categories: (a) less than 9 years of school attendance, (b) from 9 to 17 years of school attendance and (c) more than 17 years of school attendance. Self-reported information on the caregiver's gender and type of relationship with the care recipient was also obtained. The global cognitive status of the patients was assessed using the Spanish version ${ }^{12}$ of the Mini-Mental State Examination (MMSE). ${ }^{13}$ The MMSE is composed of 11 items that generate a score ranging from 0 to 30, with lower scores indicating a higher degree of cognitive impairment. Scores were adjusted for age and level of education and the cut-off score in our study was $24 / 25$ (a score below 24 indicates dementia). ${ }^{12}$

The Spanish validated version ${ }^{14}$ of the Neuropsychiatric Inventory (NPI) ${ }^{2 ; 3}$ was administered by a geriatrician to assess neuropsychiatric symptoms during a structured interview. This instrument is one of the most extensively used methods to assess NPS and it has been validated in several languages. ${ }^{15}$ In the formal care-setting, the NPI was administered to the professional caregiver (nursing assistant) while in the informal care-setting it was administered to the primary informal caregiver or the family member involved in the daily care of the patient. The caregivers rated the frequency of the symptoms using scores from 1 (occasionally, less than once per week) to 4 (very frequently, once or more per day or continuously), and their severity using scores from 1 (mild) to 3 (severe). A symptom intensity score was calculated for each neuropsychiatric symptom by multiplying its frequency (range 0-4) and severity (range 0-3). The total NPI score was 
computed by adding all composite scores of each domain (range between 0 and 144, with higher values indicating more behavioral and psychological alterations).

When the caregivers had rated the frequency and severity of each individual behavior, the Neuropsychiatric Inventory Caregiver Distress (NPI-D), ${ }^{16 ;}{ }^{17}$ was used to evaluate their own distress. They were asked to rate their emotional or psychological distress in relation to the patients individual NPS, on a scale from 0 (not at all distressing) to 5 (very severely or extremely distressing). The NPI-D provides a reliable and valid measure of subjective caregiver distress in relation to NPS measured by the NPI, and it has been shown to be useful in both clinical and research settings for assessing the contribution of NPS to caregiver distress in dementia patients. ${ }^{16}$ The NPI-D total score is the sum of individual symptom scores and ranges from 0 to 60.

\section{Statistical analysis}

The software package IBM SPSS Statistics v.23.0 was used for data analysis. Descriptive statistics (means, standard deviations, and percentages) were used to characterize the sample. In addition to this analysis, patient and caregiver's sociodemographic characteristics, as well as cognitive status, total NPI, and total NPI-D scores were compared between care-settings (formal vs. informal) through chi-square for qualitative variables and Student $t$-test for continuous variables. Pearson's correlations were calculated to measure the level of association between the intensity (frequency $\times$ severity) of NPS and caregiver distress. All NPS that showed significant correlations with NPI-D were then entered into a forward, multiple linear regression analysis to determine the best predictors of caregiving distress. Control variables referred to characteristics of patients were not included in the regression models because no significant differences were observed between the care-settings. The mean and standard deviation of the residual values were examined to ensure the accuracy of the model (mean $=.00 \pm 1.00$ ). Furthermore, the Durbin and Watson (1951) test ${ }^{18}$ was applied to examine the independence of the residuals (value 2 for completely independent). For all the analysis, the probability level considered to indicate statistical significance was set at $p<.05$. Cohen's $w$ values were reported as indicators of effect sizes (ES) ${ }^{19}$ and calculated as the square root of the ratio of the square of Pearson's contingency coefficient divided by one minus that square. We interpreted the importance of the ES using the benchmarks for 'small ES' $(w=.1)$, 'medium ES' $(w=.3)$ and 'large ES' $(w=.5)$ for measuring the distance between unpaired proportions.

\section{Results}

A total of 72 dyads of patients and their caregivers participated in the study. Table 1 shows the sociodemographic characteristics, mean MMSE scores, and NPI total scores of the patients, and gender, consanguinity percentages and total NPI-D scores of the caregivers as a function of the care-setting. There were no significant differences between formal care-setting $(n=33)$ and informal care-setting $(n=39)$ patients in mean age $(p=.435)$, gender distribution $(p=.172)$, level of education $(p=.809)$, MMSE score $(p=.746)$, and NPI total score $(p=.541)$. The mean age of the formal care-setting patients was $84.27 \pm 7.93$ years, $78.8 \%$ were female, and $84.8 \%$ had completed less than 9 years of formal education. The mean age of the informal care-setting patients was $82.79 \pm 7.99$ years, $64.1 \%$ were female, and $89.7 \%$ had completed less than 9 years of education. The mean MMSE score adjusted by age and level of education in the formal caresetting patients was $13.67 \pm 9.94$, and in the informal care-setting patients was $12.97 \pm 8.13$. Regarding the NPI score, the total NPI (frequency $\times$ severity) mean score was $13.67 \pm 9.94$ for patients of formal care-setting and $12.97 \pm 8.13$ for patients of informal care-setting. There were significant differences between the type of care-setting in caregiver gender $(p<.0001$, Cohen's effect size value $w=.411$ suggested medium practical significance) and consanguinity $(p<.0001$, $w=.801$, large practical significance). Caregivers were mostly female $(83.3 \%), 100 \%$ in the formal care-setting and $69.2 \%$ in the informal care-setting. Regarding the type of relationship, as 
expected, none of the formal caregivers had consanguinity relationship with the patient, and $79.5 \%$ of the informal caregivers had a relationship of consanguinity with the care recipient $(74.4 \%$ were sons or daughters and $5.3 \%$ were brothers or sisters, with just $15.8 \%$ being the spouses). Finally, our data showed no significant differences in NPI-D total score between the two caregiving groups $(p=.921$, the mean total distress score was $8.33 \pm 9.42$ for formal caregivers and $8.15 \pm 5.64$ for informal caregivers).

Table 1. Characteristics of patients and caregivers.

\begin{tabular}{llllll}
\hline & Total $(n=72)$ & $\begin{array}{l}\text { Formal care- } \\
\text { setting }(n=33)\end{array}$ & $\begin{array}{l}\text { Informal care- } \\
\text { setting }(n=39)\end{array}$ & $p$-value & $\begin{array}{l}\text { Effect size } \\
\text { (Cohen's } w)\end{array}$ \\
\hline Patients & & & & & \\
Age (years, mean \pm SD) & $83.47 \pm 7.94$ & $84.27 \pm 7.93$ & $82.79 \pm 7.99$ & .435 & - \\
Female, $n(\%)$ & $51(70.8 \%)$ & $26(78.8 \%)$ & $25(64.1 \%)$ & .172 & - \\
Education, $n(\%)$ & & & & .809 & - \\
$<9$ years & $63(87.5 \%)$ & $28(84.8 \%)$ & $35(89.7 \%)$ & & \\
9-17 years & $7(9.7 \%)$ & $4(12.1 \%)$ & $3(7.7 \%)$ & & \\
$>17$ years & $2(2.8 \%)$ & $1(3.0 \%)$ & $1(2.6 \%)$ & & - \\
MMSE, mean \pm SD & $13.29 \pm 8.95$ & $13.67 \pm 9.94$ & $12.97 \pm 8.13$ & .746 & - \\
Total NPI, mean $\pm \mathrm{SD}$ & $20.42 \pm 17.25$ & $21.85 \pm 22.20$ & $19.21 \pm 11.72$ & .541 & - \\
Caregivers & & & & & \\
Female, $n(\%)$ & $60(83.3 \%)$ & $33(100.0 \%)$ & $27(69.2 \%)$ & $<.0001 *$ & .411 \\
Consanguinity, $n(\%)$ & $31(43.1 \%)$ & $0(.0 \%)$ & $31(79.5 \%)$ & $<.0001 *$ & .801 \\
Total NPI-D, mean $\pm \mathrm{SD}$ & $8.24 \pm 7.55$ & $8.33 \pm 9.42$ & $8.15 \pm 5.64$ & .921 & - \\
& & & & & \\
\hline
\end{tabular}

${ }^{*} p<.05,\left(t\right.$-test for means and $\chi^{2}$ for percentages)

Abbreviations: MMSE: Mini-Mental State Examination; NPI: Neuropsychiatric Inventory; NPI-D: Neuropsychiatric Inventory Caregiver Distress Scale.

The prevalence of individual NPI symptoms is shown in Table 2 as a function of the caresetting. A bar chart showing the prevalence of individual NPS ranked by frequency is shown in Fig. 1 in both care groups. In the total sample, the most prevalent symptoms were aberrant motor behavior $(44.4 \%)$, apathy/indifference $(37.5 \%)$, agitation/aggression (33.3\%), anxiety (30.6\%), and depression/dysphoria $(27.8 \%)$, and the less prevalent symptom was elation/euphoria (12.5\%). In the formal care-setting, the most prevalent symptoms were agitation/aggression and depression/dysphoria (both 42.4\%), while in the informal care-setting the most prevalent symptoms were aberrant motor behavior (59.0\%), anxiety and apathy/indifference (both $35.9 \%$ ) (Fig. 1). Importantly, we found significant differences in the prevalence of four NPI items between the groups. While changes in appetite and eating behaviors, depression/dysphoria and irritability/lability were more prevalent in formal caregiving $(p<.0001, w=.411 ; p=.011$, $w=.301 ; p=.021, w=.271$; respectively; all representing medium practical significance), aberrant motor behavior was more prevalent in the informal caregiving context than in the formal care-setting ( $p=.007, w=.318$, medium practical significance). 
Table 2. Prevalence of NPI symptoms groups as a function of the care-setting.

\begin{tabular}{|c|c|c|c|c|c|}
\hline & Total $(n=72)$ & $\begin{array}{l}\text { Formal care- } \\
\text { setting }(n=33)\end{array}$ & $\begin{array}{l}\text { Informal care- } \\
\text { setting }(n=39)\end{array}$ & $p$-value & $\begin{array}{l}\text { Effect size } \\
(\text { Cohen's } w)\end{array}$ \\
\hline Delusions & $19(26.4 \%)$ & $9(27.3 \%)$ & $10(25.6 \%)$ & .876 & - \\
\hline Hallucinations & $14(19.4 \%)$ & $6(18.2 \%)$ & $8(20.5 \%)$ & .803 & - \\
\hline Agitation/aggression & $24(33.3 \%)$ & $14(42.4 \%)$ & $10(25.6 \%)$ & .132 & - \\
\hline Depression/dysphoria & $20(27.8 \%)$ & $14(42.4 \%)$ & $6(15.4 \%)$ & $.011 *$ & .301 \\
\hline Anxiety & $22(30.6 \%)$ & $8(24.2 \%)$ & $14(35.9 \%)$ & .285 & - \\
\hline Elation/euphoria & $9(12.5 \%)$ & $5(15.2 \%)$ & $4(10.3 \%)$ & .531 & - \\
\hline Apathy/indifference & $27(37.5 \%)$ & $13(39.4 \%)$ & $14(35.9 \%)$ & .760 & - \\
\hline Disinhibition & $11(15.3 \%)$ & $5(15.2 \%)$ & $6(15.4 \%)$ & .978 & - \\
\hline Irritability/lability & $19(26.4 \%)$ & $13(39.4 \%)$ & $6(15.4 \%)$ & $.021 *$ & .271 \\
\hline Aberrant motor behavior & $32(44.4 \%)$ & $9(27.3 \%)$ & $23(59.0 \%)$ & $.007 * *$ & .318 \\
\hline Sleep and nighttime behavior disorders & $12(16.7 \%)$ & $7(21.2 \%)$ & $5(12.8 \%)$ & .341 & - \\
\hline Appetite and eating disorders & $12(16.7 \%)$ & $11(33.3 \%)$ & $1(2.6 \%)$ & $<.0001 * *$ & .411 \\
\hline
\end{tabular}

$* p<.05 ; * * p<.001\left(\chi^{2}\right.$ test $)$.

FORMAL CARE-SETTING


Fig. 1. Prevalence of individual neuropsychiatric symptoms as a function of the caresetting (formal vs. informal). 
The correlations between mean scores in the NPI (frequency $\times$ severity) and caregivers' NPI distress scores are shown in Table 3 as a function of the care-setting. The frequency $\times$ severity scores for depression/dysphoria and changes in appetite and eating behavior symptoms were significantly higher in the formal care-setting ( $p=.038$ and $p=.001$, respectively) while the score in aberrant motor behavior was higher in the informal care-setting $(p=.014)$. In the formal caresetting, the individual symptoms with higher frequency $\times$ severity scores (most frequent and with higher severity score) were apathy/indifference $(2.91 \pm 4.35)$, appetite and eating disorders $(2.58 \pm 4.00)$, agitation/aggression $(2.55 \pm 3.64)$ and irritability/lability $(2.36 \pm 3.94)$ (see Fig. 2A). These professional caregivers showed the highest NPI distress scores for the same individual symptoms but in different order, being the more distressing symptom in this care-setting agitation/aggression (1.03 \pm 1.43$)$, followed by appetite and eating disorders $(.97 \pm 1.61)$, apathy/indifference $(.97 \pm 1.51)$, and irritability/lability $(.94 \pm 1.50)$ (see Fig. 2A). In the informal care-setting, the most intense (higher frequency $\times$ severity scores) individual symptoms were aberrant motor behavior $(4.74 \pm 4.94)$, apathy/indifference $(2.31 \pm 3.66)$, and anxiety $(2.10 \pm 3.21)$ (see Fig. 2B). Informal caregivers showed the highest NPI distress scores for the same symptoms but in different order, being the most distressing symptoms aberrant motor behavior $(1.67 \pm 1.71)$, anxiety (1.08 \pm 1.46$)$, and apathy/indifference $(.97 \pm 1.41)$ (see Fig. 2B). Elation/euphoria produced relatively little distress in both groups $(.24 \pm .75$ in formal caregivers, $.21 \pm .80$ in informal caregivers). Professional caregivers were significantly more distressed than family caregivers by appetite and eating disorders $(.97 \pm 1.61$ vs. $.05 \pm .32, p=.003)$, and family caregivers were significantly more distressed than formal caregivers by aberrant motor behavior ( $82 \pm 1.55$ vs. $1.67 \pm 1.71, p=.032$ ). As can be seen in the Table 3, correlations values indicated that all of the individual symptoms (frequency $\times$ severity scores) showed a statistically significant positive correlation with the caregiver distress in both care-settings.

Table 3. Correlation between scores in NPI (frequency $\times$ severity) and caregiver NPI distress as a function of the caresetting.

\begin{tabular}{|c|c|c|c|c|c|c|}
\hline \multirow[b]{2}{*}{ NPI symptoms } & \multicolumn{3}{|l|}{ Formal care-setting } & \multicolumn{3}{|l|}{ Informal care-setting } \\
\hline & $\begin{array}{l}\text { NPI } \\
(\text { frequency } \times \text { severity) } \\
\text { mean } \pm \text { SD }\end{array}$ & $\begin{array}{l}\text { NPI } \\
(\text { distress }) \\
\text { mean } \pm \text { SD }\end{array}$ & $r$ & $\begin{array}{l}\text { NPI } \\
(\text { frequency } \times \text { severity) } \\
\text { mean } \pm \text { SD }\end{array}$ & $\begin{array}{l}\text { NPI } \\
(\text { distress }) \\
\text { mean } \pm \text { SD }\end{array}$ & $r$ \\
\hline Delusions & $1.61 \pm 3.37$ & $.79 \pm 1.62$ & $.741 * *$ & $2.00 \pm 3.70$ & $.92 \pm 1.68$ & $.951 * *$ \\
\hline Hallucinations & $1.03 \pm 2.73$ & $.45 \pm 1.09$ & $.927 * *$ & $1.64 \pm 3.67$ & $.74 \pm 1.53$ & $.937 * *$ \\
\hline Agitation/aggression & $2.55 \pm 3.64$ & $1.03 \pm 1.43$ & $.900 * *$ & $1.87 \pm 3.64$ & $.95 \pm 1.72$ & $.961 * *$ \\
\hline Depression/dysphoria & $1.85 \pm 2.95$ & $.64 \pm 1.22$ & $.827^{* *}$ & $.62 \pm 1.66$ & $.33 \pm .93$ & $.955^{* *}$ \\
\hline Anxiety & $1.70 \pm 3.39$ & $.67 \pm 1.34$ & $.832 * *$ & $2.10 \pm 3.21$ & $1.08 \pm 1.46$ & $.819 * *$ \\
\hline Elation/euphoria & $.88 \pm 2.29$ & $.24 \pm .75$ & $.841^{* *}$ & $.56 \pm 1.98$ & $.21 \pm .80$ & $.936^{* *}$ \\
\hline Apathy/indifference & $2.91 \pm 4.35$ & $.97 \pm 1.51$ & $.960 * *$ & $2.31 \pm 3.66$ & $.97 \pm 1.41$ & $.780 * *$ \\
\hline Disinhibition & $.88 \pm 2.64$ & $.27 \pm .98$ & $.969 * *$ & $1.18 \pm 3.17$ & $.44 \pm 1.25$ & $.968^{* *}$ \\
\hline Irritability/lability & $2.36 \pm 3.94$ & $.94 \pm 1.50$ & $.944 * *$ & $1.10 \pm 2.85$ & $.51 \pm 1.30$ & $.996^{* * *}$ \\
\hline $\begin{array}{l}\text { Aberrant motor } \\
\text { behavior }\end{array}$ & $2.09 \pm 3.97$ & $.82 \pm 1.55$ & $.962 * *$ & $4.74 \pm 4.94$ & $1.67 \pm 1.71$ & $.876^{* *}$ \\
\hline $\begin{array}{l}\text { Sleep and nighttime } \\
\text { behavior disorders }\end{array}$ & $1.42 \pm 3.13$ & $.58 \pm 1.28$ & $.938 * *$ & $1.03 \pm 2.91$ & $.28 \pm .89$ & $.967 * *$ \\
\hline $\begin{array}{l}\text { Appetite and eating } \\
\text { disorders }\end{array}$ & $2.58 \pm 4.00$ & $.97 \pm 1.61$ & $.915^{* *}$ & $.05 \pm .32$ & $.05 \pm .32$ & $1.000 * *$ \\
\hline
\end{tabular}

$* p<.05 ; * * p<.001$ 


\section{A FORMAL CARE-SETTING}
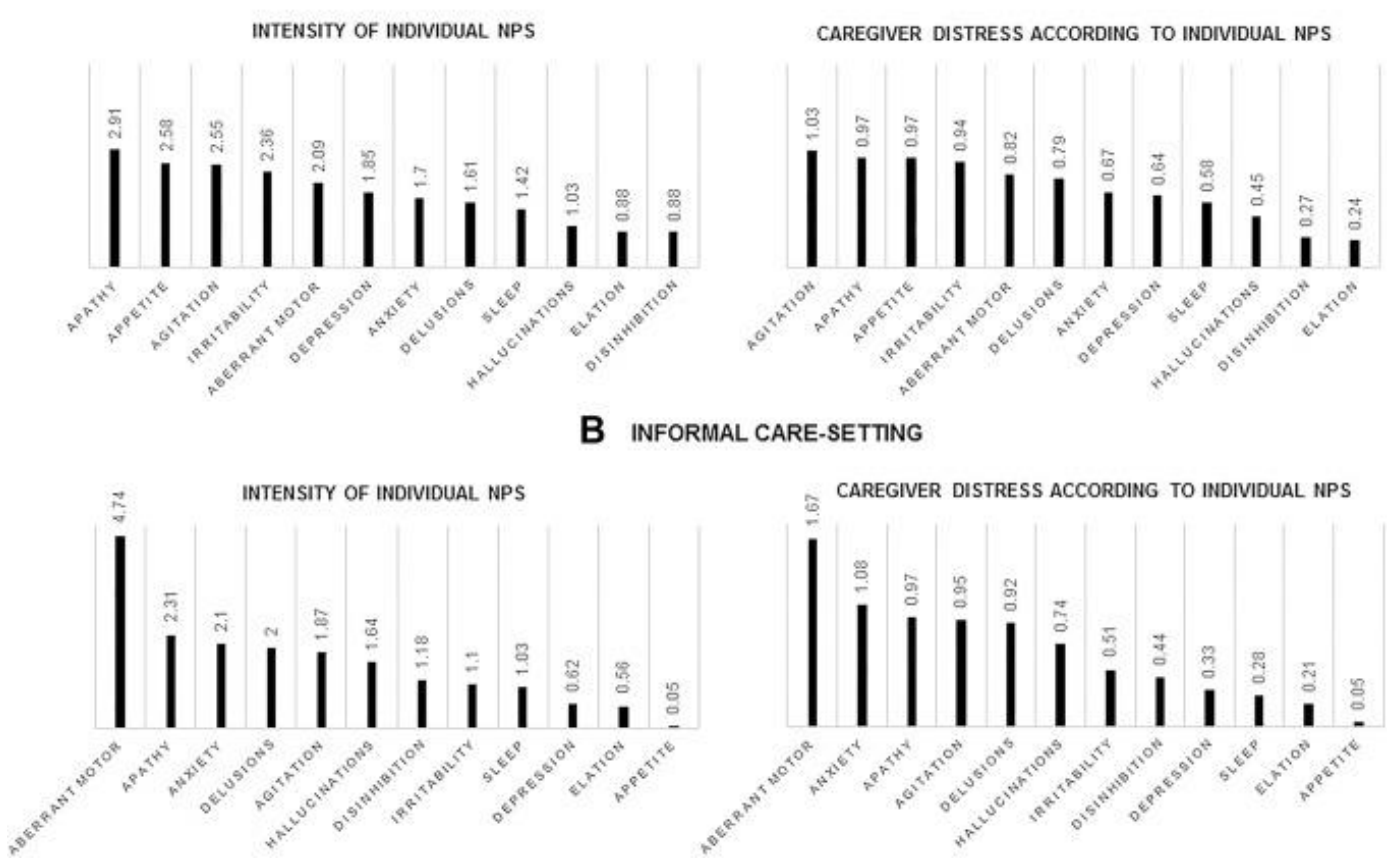

Fig. 2. Neuropsychiatric symptoms (NPS) ranked by intensity (frequency $\times$ severity) and distress ratings in both care settings. (A) Formal care-setting. (B) Informal care-setting.

To investigate the degree to which individual patients symptoms (frequency $\times$ severity scores) predicts caregivers distress (NPI-D scores), a forward multiple lineal regression was performed in both care-settings (Table 4). All the regression analysis showed good residual indexes (mean $=.00 \pm 1.00)$ and Durbin-Watson test values near 2. No multicollinearity was observed. The values for Variance Inflation Factor (VIF) for the independent variables were between 1.136 and 1.975 for formal care-setting and between 1.025 and 1.086 for informal care-setting. Tolerance values were between .506 and .881 for formal care-setting and between .921 and .976 for informal care-setting. Values of VIF lower than 10 or values of tolerance larger than .1 imply no multicollinearity diagnostic. ${ }^{20 ; 21}$ The condition number $^{22}$ was also very low, between 1.489 and 3.162 for formal care-setting and between 1.497 and 3.003 for informal care-setting, indicating that there were no multicollinearity problems. In the formal care-setting, a significant regression equation was found $(F(4,28)=43.29, p<.0001)$. The multiple correlation coefficient was .93 , indicating that approximately $84.1 \%$ of the variance of the caregiver total distress could be accounted by high patients' scores in hallucinations, sleep and night-time disturbances, anxiety, and aberrant motor behavior (see $\beta$ values in Table 4). In the informal care-setting $(F(6,32)=30.05, \quad p<.0001)$, the multiple correlation coefficient was .92 , indicating that approximately $82.1 \%$ of the variance of the caregiver total distress could be accounted by agitation/aggression, delusions, disinhibition, apathy/indifference, depression/dysphoria, and elation/euphoria. 
Table 4. Multiple linear regression analysis between neuropsychiatric symptoms (NPS) and neuropsychiatric caregiver distress (NPI-D) by group (informal vs. formal care-setting).

\begin{tabular}{|c|c|c|}
\hline \multirow{3}{*}{ Model predictor variable entered } & Formal care-setting & Informal care-setting \\
\hline & $\begin{array}{l}F_{4,28}=43.29, p<.0001, \text { adjusted } \\
R^{2}=.841\end{array}$ & $\begin{array}{l}F_{6,32}=30.05, p<.0001, \text { adjusted } \\
R^{2}=.821\end{array}$ \\
\hline & $\beta$ & $\beta$ \\
\hline Delusions & - & $.412 * *$ \\
\hline Hallucinations & $.491 * *$ & - \\
\hline Agitation/aggression & - & $.596 * *$ \\
\hline Depression/dysphoria & - & $.282 * *$ \\
\hline Anxiety & $.297 *$ & - \\
\hline Elation/euphoria & - & $.193 *$ \\
\hline Apathy/indifference & - & $.306^{* *}$ \\
\hline Disinhibition & - & $.352 * *$ \\
\hline Irritability/lability & - & - \\
\hline Aberrant motor behavior & $.164^{*}$ & - \\
\hline Sleep and nighttime behavior disorders & $.331 *$ & - \\
\hline Appetite and eating disorders & - & - \\
\hline
\end{tabular}

$* p<.05 ; * * p<.001$.

Abbreviations: $\beta$ : standardized beta coefficient

\section{Discussion}

The present paper evaluated the caregiver distress in response to the intensity of individual neuropsychiatric symptoms in older adults aged 65 or above as a function of the care-setting (formal vs. informal). Neuropsychiatric symptoms are usually categorized to differentiate them from cognitive symptoms that are also associated with dementia. The literature has successfully revealed that grouped together, neuropsychiatric symptoms are a source of significant caregiver distress. However, an effective intervention to improve the wellbeing of caregivers should examine how individual and specific patients' symptoms influence their own distress. This is important since most prevalent symptoms are not necessarily the most distressing. To the best of our knowledge, there are only a few articles that have examined the impact of individual behavioral symptoms on the caregiver distress. ${ }^{11}$ In the current exploratory study, we go a step further examining the predictability of individual neuropsychiatric symptoms, as categorized by the NPI scale, ${ }^{2 ; 3}$ on caregiver distress, in a population of formal (professional) and informal caregivers, providing a comparative study, often missed from literature involving NPS.

\section{Patient demographics}

There were no significant differences between formal and informal care-settings in mean age, gender distribution, and level of education of patients. Thus, the profile of the patient included in the present study was an elder person $(83.47 \pm 7.94)$, female $(70.8 \%)$, and with minimal education (less than 9 years of formal education; 87.5\%) regardless of the care context. There were no significant differences between care-settings in the MMSE scores, with both groups of patients showing cognitive impairment. Thus, patients in both study groups had homogeneous sociodemographic, educational, and cognitive status. 
Caregivers were mostly female $(83.3 \% ; 100 \%$ in the formal care-setting and $69.2 \%$ in the informal care-setting). Regarding the type of relationship, $79.5 \%$ of the informal caregivers had a relationship of consanguinity. This preponderance of female caregivers in our sample is consistent with previous studies suggesting the typical informal caregiver is a middle-aged female, usually the wife or the children of the patient. ${ }^{23}$ This agrees with the notion that females often outlive their spouses and are the ones who generally provide care. ${ }^{24}$ Another explanation which does not exclude the previous one could be that women still believe that it is a female's role to care for individuals who cannot care for themselves. ${ }^{25}$

\section{Prevalence of NPS as a function of the care-setting}

A great number of patients $(67.3 \%)$ had shown at least one neuropsychiatric symptom in the previous month. Although this percentage agrees with several investigations, ${ }^{26 ; 27 ; 28 ; 29 ; 30}$ it is not always congruent with the percentages found by others, ${ }^{15 ; 31 ; 32}$ which showed that up to $90.0 \%$ of dementia patients experience at least one symptom over the course of the disease. As already pointed out, the variability of this percentage could be largely dependent on the type of the sample (healthy elders, Alzheimer's disease patients, vascular dementia patients ...) and the setting considered (community-dwelling older adults, institutionalized patients ...). ${ }^{15}$ Also, a higher percentage of NPS could be obtained by using a less restrictive method of evaluating a specific behavioral symptom.

In our total sample, the most prevalent symptoms were aberrant motor behavior (44.4\%), apathy/indifference (37.5\%), agitation/aggression (33.3\%), anxiety (30.6\%), and depression/dysphoria (27.8\%), and the less prevalent symptom was elation/euphoria (12.5\%). The prevalence results of our study are comparable with others using the NPI scale to measure neuropsychiatric symptoms. In a large community population of elderly people, Lyketsos et $\mathrm{al}^{30}$ reported that the most prevalent symptoms were apathy (27\%), followed by depression (24\%), and agitation/aggression (24\%), being these behaviors four times more common in patients with dementia than in healthy older adults. Khoo et $\mathrm{al}^{7}$ found that the most common symptoms in Asian dementia patients were agitation (63.1\%), apathy (61.8\%), and night-time behavior (56.4\%), followed by depression and irritability symptoms (both 55.5\%) and that the less common symptom was elation (14.2\%). In dementia cases in rural Tanzania, the most frequent symptoms reported were anxiety (47.4\%), agitation/aggression (38.5\%), night-time behavioral disturbance (34.6\%), irritability $(33.3 \%)$ and depression $(33.3 \%){ }^{31}$ The result of the high prevalence of night-time behaviors observed in these studies contrasts with the relatively low prevalence of this symptom found in our study regardless of the care-setting $(16.7 \% ; 21.2 \%$ in the formal care-setting and $12.8 \%$ in the informal care-setting). This prevalence discrepancy might be explained by possible differences in the sample characteristics (patients with different dementia subtypes vs. subjects with different pathologies), or by ethnic and cultural differences. ${ }^{33}$

When taking into account the care context, in the formal care-setting the most prevalent symptoms were agitation/aggression and depression/dysphoria (42.4\%), followed by apathy/indifference $(39.4 \%)$. A similar result was found by Song and $\mathrm{Ho}^{34}$ in six South Korean nursing homes (agitation/aggression 52.8\%, depression/dysphoria 46.6\%). In the informal caresetting, the most prevalent symptoms were aberrant motor behavior (59.0\%), anxiety and apathy/indifference (both $35.9 \%$ ). The prevalence of aberrant motor behavior in our sample of informal patient-caregiver dyads was comparable to that found by Truzzi et al ${ }^{35}$ in a study evaluating the distress related to individual NPS among Brazilian informal caregivers, and showing that aberrant motor behavior was the second most common symptom (apathy 78.6\%, aberrant motor behavior $45.9 \%$, agitation $44.0 \%$ ). Apathy/indifference was also the most common symptom $(70.0 \%)$, and aberrant motor behavior had the highest mean NPI score in a previous study with dementia patients living in the Japanese community. ${ }^{36}$ In line with our results, apathy $(62.0 \%)$ and aberrant motor behavior $(38.0 \%)$ were the most frequent symptoms in a previous study with Brazilian Alzheimer's patients and their informal caregivers. ${ }^{37}$ In Taiwanese dementia 
patients from a memory clinic, anxiety $(37.5 \%)$ and apathy/indifference $(36.4 \%)$ were also common symptoms, ${ }^{38}$ and in Chinese dementia patients, the most common individual NPS were apathy/indifference $(79.6 \%)$, depression/dysphoria $(68.4 \%)$ and night-time behaviors $(59.2 \%) .{ }^{32}$ As a whole, apathy/indifference has shown to be one of the most prevalent NPS in informal caresettings in previous studies. ${ }^{32 ; 36 ; 37 ; 38 ; 39}$

Importantly, four NPI individual domains showed significant prevalence differences between the care-settings in the present study. Specifically, while changes in appetite and eating behaviors, depression/dysphoria and irritability/lability were more prevalent in formal caregiving, aberrant motor behavior was more prevalent in the informal caregiving context than in the formal caresetting.

\section{Correlation between the intensity of NPS and caregiver distress as a function of the care-setting}

We have studied prevalence, but also the relation between intensity (frequency $\times$ severity) of the individual NPS and the caregiver distress as a function of the care-setting. As previously stated, the total frequency $\times$ severity scores and the total distress score, when summed across all the individual symptoms, did not differed between care-settings. The fact that patients of informal care-setting were semi-institutionalized at a day-care center may have alleviated the total distress of informal caregivers in our study. However, interesting differences emerge when focusing in the presentation of specific symptoms.

In order to investigate what relationship exist between the intensity of individual NPS and formal/informal caregiver distress correlations were computed. Results showed that the intensity of individual behavioral manifestations assessed by the NPI was associated with a high level of caregiver distress in both care-settings. Research on this topic has consistently found correlations between NPS and distress on caregivers. ${ }^{11}$ When considering individual symptoms, we should be aware that there is still a lack of consistency in the approach and definition of these individual NPS which could limit the ability of researchers to clearly identify which of them have a major impact on caregiver distress. It has been also shown that caregivers who work in specialized clinics and who are experts in the diagnosis and treatment of geriatric disorders might manifest different and/or more severe problems ${ }^{16}$ than caregivers who live in the patient's immediate community. ${ }^{36}$ Moreover, it could be also possible that different cultural attitudes can influence the interpretation of NPS by caregivers. ${ }^{33}$

The most distressful NPS for professional caregivers in our study were agitation/aggression, apathy/indifference, appetite and eating disorders, and irritability/lability, and the less distressful was elation/euphoria. According to this finding, agitation/aggression was the most distressing symptom and elation/euphoria was the less distressing symptom for nurses and care workers of nursing homes in previous studies. ${ }^{40}$

Aberrant motor behavior, anxiety, and apathy/indifference were the most distressful NPS according to our sample of informal caregivers. Aberrant motor behavior, mainly consisting in wandering and repetitive purposeless or inappropriate activities, was previously reported as one of the most distressful symptoms for informal caregivers, possibly because it imposes high physical demands to the caregiver. ${ }^{35}$ According to our results, anxiety has been also shown as one of the most distressing symptoms for Taiwanese informal caregivers, ${ }^{38}$ and apathy/indifference caused the highest level of distress followed by agitation and aggression for Brazilian informal caregivers. ${ }^{37}$ Apathy/indifference symptom was also rated as one of the most distressing symptoms for Chinese informal caregivers, together with delusions and hallucinations. ${ }^{32}$ In a sample of dementia patients not referred to a clinic, the behavioral disturbances that most frequently increased the informal caregiver distress were aggression, wandering, and incontinence. ${ }^{41}$ The study of Matsumoto et al, ${ }^{36}$ enrolling elderly dementia patients living with their caregivers in the community, showed that agitation/aggression, delusions, and irritability/lability were the 
symptoms that correlate the most with informal caregiver distress, although their frequency and severity were low.

Because of their intensity and adverse effects on the caregivers, the early recognition, and management of these specific patients' symptoms may lead to better care and quality of life for patients and formal/informal caregivers.

Importantly, the most intense (most frequent and severe) symptoms manifested by the patients in formal and informal caregiving contexts were also the ones causing more caregiver distress in our study. This result is in contrast with others $36 ; 38 ; 39$ indicating that caregiver distress does not necessarily depend on frequency and severity of the NPS. Given the different results, we propose to educate caregivers on how to deal and handle with the most distressful behaviors independently if they are or not the most frequent or severe ones. While music therapy, multisensory stimulation therapy, or therapeutic touch are good examples of interventions to improve NPS in an informal care context, behavioral management techniques are very effective for reduction of NPS in a formal caregiving context. ${ }^{42}$ A recent systematic review focused on the management of agitation in Alzheimer's disease patients has revealed that interactive music therapy is an optimal nonpharmacological intervention for institutionalized patients. ${ }^{43}$ The review also highlights the need of future evidence on the effectiveness of non-pharmacological therapies in non-institutionalized $\mathrm{AD}$ patients and the long-term effects of these interventions. ${ }^{43}$ Recent results suggest that multisensory stimulation could have even better effects on anxiety symptoms and dementia severity than individualized music sessions in elderly patients with severe dementia. ${ }^{44}$

In some previous studies, groups of similar or correlated neuropsychiatric symptoms have been studied together into clusters or factors (see van der Linde et $\mathrm{al}^{45}$ for a review), indicating that these groups may differ in their associations with cognitive function, natural course, caregiver burden, most effective treatment and underlying biology or etiology. In the present paper, and given the large individual variability of the symptoms, we have however studied each single symptom individually to further explore potential clinical differences as a function of the caresetting.

To our knowledge, this is the first exploratory study to investigate the degree to which the intensity of individual patients' symptoms predicts caregivers' distress as a function of the caresetting (formal/professional vs. informal). Taking into account that the practices and expectations of the professional and informal family care systems are greatly dissimilar (rational and decontextualized vs. emotional and contextualized, respectively ${ }^{46}$ this is a relevant point. In the formal care-setting, high patients' scores in hallucinations, sleep and night-time disturbances, anxiety, and aberrant motor behavior predicted a high percentage of caregiver total distress, while in the informal care-setting the best predictors of caregiver distress were high scores in agitation/aggression, delusions, disinhibition, apathy/indifference, depression/dysphoria, and elation/euphoria. These data confirm the influence of the care-setting when trying to predict the caregiving distress or burden through the NPS. Results appear to be also consistent with clinical and real life observations, where the most distressing symptoms for informal family caregivers are the most emotional ones while the symptoms that predict more distress in professional caregivers are the ones that interfere the most with his/her professional duties at nursing homes. Given the modest efficacy of current non-pharmacological and pharmacological strategies used for managing NPS, ${ }^{15 ; 47}$ and taking into account that dementia patients are highly dependent on informal caregivers - possibly leading to a premature nursing home placement, ${ }^{8}$ and mainly based on our findings implicating a large amount of individual symptoms predicting distress in formal and informal caregiving, we propose that it should be convenient to create more educational interventions to help the family members and professionals to deal with NPS and their own distress. We also suggest the definition of specific interventions when it comes to create programs that address NPS in a specific caregiving context, thus avoiding the significant financial pressures these symptoms generates on caregivers. 
The present study presents some limitations. Due to its cross-sectional nature, we could not examine the timing of neuropsychiatric symptoms over the course of the patient-caregiver relationship, which may be important in understanding the correlation between each individual symptom and the caregiver distress. Finally, the present study did not investigate factors related to patient's medication. One of the strengths of the study is that neuropsychiatric symptoms were addressed individually and not as a global psychiatric symptomatology (total NPI score). This individualized method of acquiring and analyzing the results could help experts to obtain information about which symptoms should be treated prior to the beginning of the care depending on whether the caregiving is formal or informal. Further studies involving larger samples of individuals with different dementia illnesses are necessary to study the impact of each behavioral symptom and the type of dementia as a function of the caregiver-setting (formal vs. informal). Because certain intrinsic caregivers' characteristics, independent of care recipients' characteristics, have been associated with the presence of NPS $^{46}$ and caregiver distress, ${ }^{48}$ associations and dynamics between them should be also further investigated.

Finally, it is important to note that in the present study the sample was rather homogeneous in terms of race/ethnicity (Western Europeans, white race) and culture. Potential cross-cultural differences in the way caregivers interact with patients may exist, ${ }^{33 ;} 35$ and recommended treatments may not have the same efficacy among different cultures. ${ }^{7}$ Thus, it should be interesting to replicate the present study using more heterogeneous samples with diverse ethnic and cultural backgrounds. Knowledge of potential differences, which may affect the caregivers' response to specific symptoms, is essential to elaborate more culture specific treatments.

Because reducing caregivers burden is a social problem in an aging society, we hope that our contribution will help both professional and family caregivers develop further strategies and symptoms-targeted interventions for the benefit of dementia older adults.

\section{Conclusion}

Neuropsychiatric symptoms are widely associated with global outcomes for caregivers, specifically with caregiver distress, increasing the health care costs and also the risk of institutionalization of the elderly. Our results highlight the importance of identifying which individual NPS predicts distress in caregivers, depending on the care-setting (formal vs. informal). A timely identification could help experts in targeting effectively the interventions. As neuropsychiatric problems are potentially treatable or at least ameliorated with therapy, these population-based findings are important to devise effective methods for improving future interventions. This might help in the choice of specific treatment modalities to reduce the caregiver burden depending on the context and thus, reduce the public health spending.

\section{References}

1. European Comission. The 2015 Aging Report. Underlying Assumptions and Projection Methodologies. (2014) http://dx.doi.org/10.2765/76255.

2. J.L. Cummings, M. Mega, K. Gray, S. Rosenberg-Thompson, D.A. Carusi, J. Gornbein. The neuropsychiatric inventory: comprehensive assessment of psychopathology in dementia. Neurology, 44 (12) (1994), pp. 2308-2314 http://dx.doi.org/10.1212/WNL.44.12.2308.

3. J.L. Cummings. The neuropsychiatric inventory: assessing psychopathology in dementia patients. Neurology, 48 (5 suppl 6) (1997), pp. S10-S16 http://dx.doi.org/10.1212/WNL.48.5_Suppl_6.10S.

4. J.C. Millán-Calenti, M. Gandoy-Crego, M. Antelo-Martelo, M. López-Martínez, M.P. Riveiro-López, J.M. Mayán-Santos. Helping the family carers of Alzheimer's patients: from theory to practice. A preliminary study. Arch Gerontol Geriatr, 30 (2) (2000), pp. 131-138 http://dx.doi.org/10.1016/S01674943(00)00044-3.

5. P.V. Rabins, N.L. Mace, M.J. Lucas. The impact of dementia on the family. JAMA, 248 (3) (1982), pp. 333-335 http://dx.doi.org/10.1001/jama.1982.03330030039022. 
6. K. Warchol-Biedermann, E. Mojs, R. Gregersen, K. Maibom, J.C. Millán-Calenti, A. Maseda. What causes grief in dementia caregivers?. Arch Gerontol Geriatr, 59 (2) (2014), pp. 462-467 http://dx.doi.org/10.1016/j.archger.2014.05.013.

7. S.A. Khoo, T.Y. Chen, Y.H. Ang, P. Yap. The impact of neuropsychiatric symptoms on caregiver distress and quality of life in persons with dementia in an Asian tertiary hospital memory clinic. Int Psychogeriatr, 25 (12) (2013), pp. 1991-1999 http://dx.doi.org/10.1017/S1041610213001518.

8. B.F. O'Donnell, D.A. Drachman, H.J. Barnes, K.E. Peterson, J.M. Swearer, R.A. Lew. Incontinence and troublesome behaviors predict institutionalization in dementia. J Geriatr Psychiatry Neurol, 5 (1) (1992), pp. 45-52 http://dx.doi.org/10.1177/002383099200500108.

9. E. Mossello, V. Caleri, E. Razzi, et al. Day care for older dementia patients: favorable effects on behavioral and psychological symptoms and caregiver stress. Int J Geriatr Psychiatry, 23 (10) (2008), pp. 1066-1072 http://dx.doi.org/10.1002/gps.2034.

10. Y. Miyamoto, H. Tachimori, H. Ito. Formal caregiver burden in dementia: impact of behavioral and psychological symptoms of dementia and activities of daily living. Geriatr Nurs, 31 (4) (2010), pp. 246253 http://dx.doi.org/10.1016/j.gerinurse.2010.01.002.

11. K. Ornstein, J.E. Gaugler. The problem with "problem behaviors": a systematic review of the association between individual patient behavioral and psychological symptoms and caregiver depression and burden within the dementia patient-caregiver dyad. Int Psychogeriatr, 24 (10) (2012), pp. 1536-1552 http://dx.doi.org/10.1017/S1041610212000737.

12. R. Blesa, M. Pujol, M. Aguilar, et al. Clinical validity of the 'mini-mental state' for Spanish speaking communities. Neuropsychologia, 39 (11) (2011), pp. 1150-1157 http://dx.doi.org/10.1016/S00283932(01)00055-0.

13. M.F. Folstein, S.E. Folstein, P.R. McHugh. "Mini-mental state". A practical method for grading the cognitive state of patients for the clinician. J Psychiatr Res, 12 (3) (1975), pp. 189-198 http://dx.doi.org/10.1016/0022-3956(75)90026-6.

14. J. Vilalta-Franch, M. Lozano-Gallego, M. Hernández-Ferrándiz, J. Llinàs-Reglà, S. López-Pousa, O.L. López. Neuropsychiatric inventory. The psychometric properties of its adaptation to Spanish. [In Spanish]. Rev Neurol, 29 (1) (1999), pp. 15-19 http://www.ncbi.nlm.nih.gov/pubmed/10528303.

15. J. Cerejeira, L. Lagarto, E.B. Mukaetova-Ladinska. Behavioral and psychological symptoms of dementia. Front Neurol, 3 (2012), p. 73 http://dx.doi.org/10.3389/fneur.2012.00073.

16. D.I. Kaufer, J.L. Cummings, D. Christine, et al. Assessing the impact of neuropsychiatric symptoms in Alzheimer's disease: the neuropsychiatric inventory caregiver distress scale. J Am Geriatr Soc, 46 (2) (1998), pp. 210-215 http://dx.doi.org/10.1111/j.1532-5415.1998.tb02542.x.

17. M. Boada, J.C. Cejudo, L. Tárraga, O.L. López, D. Kaufer. Neuropsychiatric inventory questionnaire (NPI-Q): Spanish validation of a brief clinical form of the neuropsychiatric inventory (NPI). [In Spanish]. Neurologia, 17 (6) (2002), pp. 317-323 http://www.ncbi.nlm.nih.gov/pubmed/12084358.

18. J. Durbin, G.S. Watson. Testing for serial correlation in least squares regression. II. Biometrika, 38 (1-2) (1951), pp. 159-178 http://dx.doi.org/10.1093/biomet/38.1-2.159

19. J. Cohen. Statistical Power Analysis for the Behavioural Sciences. (2nd ed.)Erlbaum, Hillsdale, NJ (1988).

20. M. Kutner, C.J. Nachtsheim, J. Neter, W. Li. Applied Linear Statistical Models. (5th ed.)McGraw-Hill Irwin, New York (2005).

21. D.N. Gujarati. Econometría Básica. (5th ed.)McGraw Hill, México (2010).

22. D.C. Montgomery, E.A. Peck. Introduction to Linear Regression Analysis. (2nd ed.)John Wiley \& Sons, New York (1992).

23. I. Contador, B. Fernández-Calvo, D.L. Palenzuela, S. Miguéis, F. Ramos. Prediction of burden in family caregivers of patients with dementia: a perspective of optimism based on generalized expectancies of control. Aging Ment Health, 16 (6) (2012), pp. 675-682 http://dx.doi.org/10.1080/13607863.2012.684666.

24. C. Donaldson, N.A. Tarrier, A. Burns. Determinants of carer stress in Alzheimer's disease. Int J Geriatr Psychiatry, 13 (4) (1998), pp. 248-256 4<248::AID-GPS770>3.0.CO;2-0 http://dx.doi.org/10.1002/(SICI)1099-1166(199804)13.

25. M.L. Friedemann, K.C. Buckwalter. Family caregiver role and burden related to gender and family relationships. J Fam Nurs, 20 (3) (2014), pp. 313-336 http://dx.doi.org/10.1177/1074840714532715

26. A. Burns, R. Jacoby, R. Levy. Psychiatric phenomena in Alzheimer's disease. I: disorders of thought content. Brit J Psychiatry, 157 (1) (1990), pp. 72-76 http://dx.doi.org/10.1192/bjp.157.1.72.

27. A. Burns, R. Jacoby, R. Levy. Psychiatric phenomena in Alzheimer's disease. II: disorders of perception. Brit J Psychiat, 157 (1) (1990), pp. 76-81 http://dx.doi.org/10.1192/bjp.157.1.76.

28. A. Burns, R. Jacoby, R. Levy. Psychiatric phenomena in Alzheimer's disease. III: disorders of mood. Brit J Psychiat, 157 (1) (1990), pp. 81-86 http://dx.doi.org/10.1192/bjp.157.1.81.

29. A. Burns, R. Jacoby, R. Levy. Psychiatric phenomena in Alzheimer's disease. IV: disorders of behaviour. Brit J Psychiat, 157 (1) (1990), pp. 86-94 http://dx.doi.org/10.1192/bjp.157.1.86. 
30. C.G. Lyketsos, M. Steinberg, J.T. Tschanz, M.C. Norton, D.C. Steffens, J.C. Breitner. Mental and behavioral disturbances in dementia: findings from the cache county study on memory in aging. Am J Psychiatry, 157 (5) (2000), pp. 708-714 http://dx.doi.org/10.1176/appi.ajp.157.5.708.

31. S.M. Paddick, A. Kisoli, A. Longdon, et al.. The prevalence and burden of behavioural and psychological symptoms of dementia in rural Tanzania. Int J Geriatr Psychiatry, 30 (8) (2014), pp. 815-823 http://dx.doi.org/10.1002/gps.4218.

32. J. Wang, L.D. Xiao, X. Li, A. De Bellis, S. Ullah. Caregiver distress and associated factors in dementia care in the community setting in China. Geriatr Nurs, 36 (5) (2015), pp. 348-354 http://dx.doi.org/10.1016/j.gerinurse.2015.04.013.

33. J.H. Flaskerud. Ethnicity, culture, and neuropsychiatry. Issues Ment Health Nurs, 21 (1) (2000), pp. 5-29 http://dx.doi.org/10.1080/016128400248248

34. J.A. Song, Y. Oh. The association between the burden on formal caregivers and behavioral and psychological symptoms of dementia (BPSD) in Korean elderly in nursing homes. Arch Psychiatr Nurs, 29 (5) (2015), pp. 346-354 http://dx.doi.org/10.1016/j.apnu.2015.06.004.

35. A. Truzzi, L. Valente, E. Engelhardt, J. Laks. The association between caregiver distress and individual neuropsychiatric symptoms of dementia. Dement Neuropsychol, 7 (3) (2013), pp. 286-291 http://www.redalyc.org/articulo.oa?id=339529044009 Accessed 13.01.2016

36. N. Matsumoto, M. Ikeda, R. Fukuhara, et al. Caregiver burden associated with behavioral and psychological symptoms of dementia in elderly people in the local community. Dement Geriatr Cogn Disord, 23 (4) (2007), pp. 219-224 http://dx.doi.org/10.1159/000099472.

37. C. Godinho, A. Camozzato, R. Kochhann, M.L. Fagundes Chaves. Association of caregiver demographic variables with neuropsychiatric symptoms in Alzheimer's disease patients for distress on the neuropsychiatric inventory (NPI). Dement Neuropsychol, 2 (3) (2008), pp. 211-216 http://www.redalyc.org/articulo.oa?id=339529003013 Accessed 13.01.2016.

38. S.S. Huang, M.C. Lee, Y.C. Liao, W.F. Wang, T.J. Lai. Caregiver burden associated with behavioral and psychological symptoms of dementia (BPSD) in Taiwanese elderly. Arch Gerontol Geriatr, 55 (1) (2012), pp. 55-59 http://dx.doi.org/10.1016/j.archger.2011.04.009.

39. E.B. Fauth, A. Gibbons. Which behavioral and psychological symptoms of dementia are the most problematic? Variability by prevalence, intensity, distress ratings, and associations with caregiver depressive symptoms. Int J Geriatr Psychiatry, 29 (3) (2014), pp. 263-271 http://dx.doi.org/10.1002/gps.4002.

40. S.A. Zwijsen, A. Kabboord, J.A. Eefsting, et al. Nurses in distress? An explorative study into the relation between distress and individual neuropsychiatric symptoms of people with dementia in nursing homes. Int J Geriatr Psychiatry, 29 (4) (2014), pp. 384-391 http://dx.doi.org/10.1002/gps.4014.

41. N. Nagaratnam, M. Lewis-Jones, D. Scott, L. Palazzi. Behavioral and psychiatric manifestations in dementia patients in a community: caregiver burden and outcome. Alzheimer Dis Assoc Disord, 12 (4) (1998), pp. 330-334 http://dx.doi.org/10.1097/00002093-199812000-00013.

42. G. Livingston, K. Johnston, C. Katona, J. Paton, C.G. Lyketsos, Old Age Task Force of the World Federation of Biological Psychiatry. Systematic review of psychological approaches to the management of neuropsychiatric symptoms of dementia. Am J Psychiatry, 162 (11) (2005), pp. 1996-2021 http://dx.doi.org/10.1176/appi.ajp.162.11.1996.

43. J.C. Millán-Calenti, L. Lorenzo-López, B. Alonso-Búa, C. de Labra, I. González-Abraldes, A. Maseda. Optimal non-pharmacological management of agitation in Alzheimer's disease: challenges and solutions. Clin Interv Aging, 11 (2016), pp. 175-184 http://dx.doi.org/10.2147/CIA.S69484.

44. A. Sánchez, A. Maseda, M.P. Marante-Moar, C. de Labra, L. Lorenzo-López, J.C. Millán-Calenti. Comparing the effects of multisensory stimulation and individualized music sessions on elderly people with severe dementia: a randomized controlled trial. J Alzheimers Dis, 52 (2016), pp. 303-315 http://dx.doi.org/10.3233/JAD-151150.

45. R.M. van der Linde, T. Dening, F.E. Matthews, C. Brayne. Grouping of behavioural and psychological symptoms of dementia. Int J Geriatr Psychiatry, 29 (6) (2014), pp. 562-568 http://dx.doi.org/10.1002/gps.4037.

46. P. Singh, R. Hussain, A. Khan, L. Irwin, R. Foskey. Dementia care: intersecting informal family care and formal care systems. J Aging Res, 2014 (2014), pp. 1-9 Article ID 486521 http://dx.doi.org/10.1155/2014/486521.

47. K.M. Sink, K.E. Covinsky, D.E. Barnes, R.J. Newcomer, K. Yaffe. Caregiver characteristics are associated with neuropsychiatric symptoms of dementia. J Am Geriatr Soc, 54 (5) (2006), pp. 796-803 http://dx.doi.org/10.1111/j.1532-5415.2006.00697.x.

48. I. González-Abraldes, J.C. Millán-Calenti, L. Lorenzo-López, A. Maseda. The influence of neuroticism and extraversion on the perceived burden of dementia caregivers: an exploratory study. Arch Gerontol Geriatr, 56 (1) (2013), pp. 91-105 http://dx.doi.org/10.1016/j.archger.2012.07.011. 